\title{
CARD9 as a potential target in cardiovascular disease
}

This article was published in the following Dove Press journal:

Drug Design, Development and Therapy

22 November 2016

Number of times this article has been viewed

\section{Matthew R Peterson \\ Samantha E Haller \\ Jun Ren \\ Sreejayan Nair \\ Guanglong $\mathrm{He}$}

School of Pharmacy, University of Wyoming, College of Health Sciences, Laramie, WY, USA
Correspondence: Guanglong He School of Pharmacy, University of Wyoming, College of Health Sciences, 1000 East University Avenue, Laramie, WY 8207I, USA

Tel +I 3077666637

Fax + I 3077662953

Email ghe@uwyo.edu
Abstract: Systemic inflammation and localized macrophage infiltration have been implicated in cardiovascular pathologies, including coronary artery disease, carotid atherosclerosis, heart failure, obesity-associated heart dysfunction, and cardiac fibrosis. Inflammation induces macrophage infiltration and activation and release of cytokines and chemokines, causing tissue dysfunction by instigating a positive feedback loop that further propagates inflammation. Cytosolic adaptor caspase recruitment domain family, member 9 (CARD9) is a protein expressed primarily by dendritic cells, neutrophils, and macrophages, in which it mediates cytokine secretion. The purpose of this review is to highlight the role of CARD9 as a potential target in inflammation-related cardiovascular pathologies.

Keywords: inflammation, heart failure, pressure-overload, hypertension

\section{Introduction}

Despite a modest, but encouraging, reduction in cardiovascular disease (CVD) in the past decade, CVD persists as a topmost cause of mortality in the US ( $30 \%)$. Novel multifaceted interventions are necessary, as a number of risk factors for CVD are common in the population. Approximately 80 million Americans have hypertension. Predictably, the percentage of deaths attributable to CVD mirrors the prevalence of hypertension, which is a leading cause for myocardial hypertrophy that may progress to heart failure and death. Hypertension is also a risk factor for stroke and ischemic heart disease. ${ }^{1,2}$ Obesity, metabolic syndrome, hypertension, dyslipidemia, and CVD are clearly interrelated comorbidities. Those with multiple comorbidities induce elevated risk and those with fewer comorbidities confer less risk. Considering these facts, interventions with a broad scope that may underlie multiple comorbidities may have the greatest benefit.

Persistent systemic inflammation has been linked to a number of cardiovascular complications. Furthermore, macrophages and neutrophils may infiltrate organs (including the myocardium) and propagate a paracrine inflammatory response through cytokine and chemokine secretion. This action is identified as a putative factor in insulin resistance, mitochondrial dysfunction, fibrosis, elevated reactive oxygen species (ROS), and a prolonged local inflammatory response..$^{3-7}$

An opportunity presents itself in investigating further the action of a dysregulated immune cell signaling in CVD. Immune cell function in vital organs is not pathological in the healthy state, but with chronic exposure to inflammatory cytokines, the tissue cells begin to display abnormal signaling. 


\section{Pattern recognition receptors (PRRs)}

PRRs of the immune system are reviewed extensively elsewhere. ${ }^{8}$ In brief, PRRs are signaling proteins that recognize molecules commonly expressed by pathogens. PRRs may recognize pathogen-associated molecular patterns (PAMPs) expressed by fungi, bacteria, viruses, and parasites. Some PAMPs can penetrate cell walls. Accordingly, some PRRs are expressed in the cytosol as opposed to on the cell membrane. Once activated, they initiate a signaling cascade to spur the immune system into action to kill and/or remove the pathogens. Generally, a PRR signal is propagated through the cell via protein-protein interactions and protein phosphorylation, the net result of which is the activation of transcription factors for protein expression of cytokines and other immune system components necessary to respond to the infection.

\section{CARD9}

Cytosolic adaptor caspase recruitment domain family, member 9 (CARD9), a scaffolding protein expressed in monocytes, neutrophils, dendritic cells, and macrophages, has been identified as a major constituent in innate immunity, acting as a transducer in several intracellular pathways and coordinating T-cell migration in response to pathogens.

CARDs typically interact with other CARD-domaincontaining proteins to transduce signals in response to the extracellular milieu to spur a coordinated intracellular response. ${ }^{9}$ While CARD9 itself does not recognize any pathogens, it provides a link between the PRRs on the cell membrane and the appropriate intracellular response.

CARD9 mediates innate immune response to numerous fungi, including zymosan, ${ }^{10}$ Candida albicans, ${ }^{11}$ and Candida tropicalis. ${ }^{12}$ Fungi and mycobacterium ${ }^{11}$ signaling pathways begin with recognition by a receptor on the extracellular side of the membrane, with C-type lectin receptors. ${ }^{13} \mathrm{C}$-type lectins (dectin-1, dectin-2, dectin-3 and mincle) are PRRs coupled with an intracellular protein spleen tyrosine kinase (SyK). ${ }^{14}$ When a C-type lectin encounters zymosan ${ }^{15}$ or C. albicans ${ }^{16}$ or another glucan chain, SyK activates protein kinase C (PKC), which phosphorylates CARD9, causing it to bind to the CARD domain of B-cell lymphoma/leukemia 10 (BCL10), ${ }^{9}$ which associates with mucosa-associated lymphoid tissue lymphoma translocation protein 1 (MALT1), forming the CARD9-BCL10-MALT1 signalosome that initiates the chemokine/cytokine response coordinated by nuclear factor- $\mathrm{\kappa B}(\mathrm{NF}-\kappa \mathrm{B}) .{ }^{10}$ This includes the accumulation of pro-interleukin (IL)-1 $\beta$, IL-1 $\beta$ 's precursor, in the cytosol.
There is evidence to suggest that the innate immune response is PRR specific. An NF- $\kappa B$ response can be coordinated by CARD9 when dectin-2 is activated, but $\mathrm{NF}-\mathrm{KB}$ upregulation is not dependent on CARD9 when the receptor dectin-1 is activated. A parallel non-traditional CARD9-dependent mechanism was concurrently introduced wherein dectin-1 signals phosphorylation of a Ras-growth-regulating factor 1 (GRF1)/CARD9/H-Ras complex by SyK that activates a tumor necrosis factor- $\alpha$ (TNF- $\alpha$ ) response through extracellular signal-regulated kinase (ERK). ${ }^{11}$

CARD9 is also involved in PRR signaling for intracellularly recognized ligands (muramyl dipeptide [MDP] and RNA). Nucleotide-binding oligomerization domain-containing protein 2 (NOD2) is an intracellular PRR that recognizes MDP, a molecular pattern expressed in bacteria. ${ }^{17}$ NOD2 complexes with CARD9 and CARD9 receptor-interacting serine-threonine kinase 2 (RICK/RIP2). ${ }^{18}$ Presence of CARD9 in the complex is required for the activation of $\mathrm{p} 38$ mitogen-activated protein kinase (p38 MAPK) and C-jun N-terminal kinase (JNK), while RICK/RIP2 must be bound for full NF- $\kappa B$ activation in response to MDP. ${ }^{19}$ Each of the three transcription factors enhance secretion of proinflammatory cytokines, particularly IL- 6 and TNF- $\alpha$.

Intracellular viral RNA initiates CARD9 signaling in a separate pathway. CARD9-BCL10 complex (absent MALT1) is recruited by mitochondrial antiviral signaling protein (MAVS) after MAVS is activated by a retinoic acidinducible gene 1 (RIG-1)-like receptor (RLR). RLRs are sensitive to the RNA of viruses. The end result is activation of NF-KB and accumulation of pro-IL-1 $\beta$ in the cytosol, allowing increased secretion of IL- $1 \beta .{ }^{20,21}$ There is also evidence that RIG-1 can also sense nucleic acids from bacteria, making CARD9 doubly important in the response to bacterial pathogens. ${ }^{22}$

Genetic abnormalities in CARD9 have been linked to immune deficiency in human beings, ${ }^{21}$ while overexpression may be involved in gastric B-cell lymphoma, ${ }^{22}$ metastasis, ${ }^{23}$ and inflammatory disorders such as Crohn's disease and inflammatory bowel disease (IBD) ${ }^{23-27}$ Dysregulated CARD9 signaling has been implicated in autoimmune diseases of the eye, ${ }^{28}$ contact hypersensitivity, ${ }^{29}$ and aseptic acute pancreatitis. ${ }^{30}$ CARD9 is a strong therapeutic candidate for multiple reasons. It is downstream from multiple PRRs, so it signals in multiple pathways. It is a non-redundant mediator of cytokine secretion and is present ubiquitously in the innate immune cells. Pathologies linked to macrophage or neutrophil-mediated inflammation - especially in the case of cytokine-related dysfunction - should be investigated 
to determine if intercession in CARD9 signaling could be therapeutic.

\section{Paracrine action of macrophages and neutrophils in CVD}

In a healthy immune system, macrophages and neutrophils propagate a necessary immune response. Recognition, phagocytosis, and degradation of pathogens and the secretion of cytokines and chemotactic factors are required for a healthy immune response. These cytokines and chemotactic factors propagate inflammatory signals in nearby tissues and the resultant changes in protein transcription, translation, and expression as well as the movement of other inflammatory cells to the local area. This has the desired effect of breakdown and removal of pathogens, infection, and damaged cells. Dysfunctional inflammatory signaling influences the progression and outcome of CVDs. A chronic low-grade systemic inflammation is linked to CVD, ${ }^{31,32}$ but a potent paracrine effect exerted by the cytokine mediators of infiltrating immune cells has also been identified. Inflammatory changes to myocardial injury are described in myocardial ischemic injury, including elevation in chemokines, ${ }^{33}$ macrophages, ${ }^{34}$ and neutrophils..${ }^{35,36}$ To what degree this response is necessary or deleterious is not always clear and may depend on the context of the disease.

Conflicting results in the modulation or augmentation of macrophage signaling in disease models exist. For instance, Morimoto et $\mathrm{al}^{37}$ reported that monocyte chemoattractant protein-1 (MCP-1) (a chemoattractant for immune cells) overexpression resulted in augmented macrophage infiltration and abrogated left ventricular dysfunction and fibrosis in infarcted mice. Frangogiannis et $\mathrm{al}^{38}$ investigated the effects of ischemia-reperfusion on MCP-1 knockout mice and concluded that MCP-1 likely drives the extent of macrophage accumulation. However, alleviating the myocardium of the macrophage infiltration resulted in a reduced fibrosis and rescued it from cardiomyopathy. The reasons for the discrepancy may be attributable to the model employed. Morimoto et al employed a chronic myocardial infarction (MI) model, whereas Frangogiannis et al utilized an acute MI with subsequent reperfusion. It is possible that macrophage infiltration and the requisite signaling are alleviative in the absence of reperfusion, but are deleterious in its presence.

A similar lack of clarity exists when studying the net effect of macrophages in hypertensive animal models. Clodronateinfused liposomes have been used to suppress macrophage presence in hypertensive rat models in order to elucidate the role of macrophage signaling. Investigators have consistently reported reduced fibrosis. The effect of macrophage depletion on myocardial function was beneficial in one study, ${ }^{39}$ but clodronate-exacerbated hypertension induced function decrements in another. ${ }^{40}$ Of course, complete deletion of macrophages is not desirable, but the concept of blunting the effects of proinflammatory macrophages is supported. Reports on the effects of macrophage deletion or signaling inhibition have been mixed, lending credence to the idea that macrophage signaling is not binary but a multi-faceted arena that offers potential and requires precise study.

\section{Cytokines in the myocardium}

Cytokine-specific outcomes on myocardial function and remodeling are difficult to parse. Absent pharmaceutical intervention, they act in concert, making laboratory results achieved by manipulating a single cytokine difficult to translate into successful therapeutic drug trials. Macrophage IL-6 induces TGF- $\beta$ signaling in fibroblasts and the ensuing fibrosis, concentric hypertrophy, collagen accumulation, and stiffness in the myocardium. ${ }^{41,42}$ Concurrently, low-grade induction of IL-1 $\beta$ triggers cardiomyocyte hypertrophy in vitro, but antagonizes fibroblast expression, perhaps driving compensatory hypertrophy in vivo by inhibiting fibrosis. ${ }^{43}$ These findings make IL-1 $\beta$ as a troubling potential target. As just discussed, a low level of IL-1 $\beta$ in the myocardium may be beneficial. However, a higher level of IL- $1 \beta$ may still cause dysfunction. IL-1 $\beta$ is a CARD9-related cytokine, so this might indicate that any protective effect of targeting CARD9 that is due in part to its effect on IL- $1 \beta$ is likely to be mediated through a blunted or rescued hypertrophic response rather than mitigating fibrosis. Alternatively, CARD9 knockout may alleviate fibrosis through a different mechanism.

Early findings would lead one to believe that any of the aforementioned macrophage-induced cytokines may be effectively targeted in CVD. CARD9 has also been linked to the cytokine IL-17, as it is requisite for differentiation of Th- 17 cells. ${ }^{44}$ IL- $17,{ }^{45}$ IL- $1 \beta,{ }^{46}$ TNF- $\alpha,{ }^{47}$ and IL- $6{ }^{42}$ in rats have separately been shown to induce cardiac dysfunction or fibrosis via injection or infusion in animals. Interestingly, knockout of IL-1 $\beta$ impaired compensatory hypertrophy in mice in a pressure-overload model ${ }^{48}$ It seems that the effect of IL- $1 \beta$ inhibition depends on the degree of inflammation, as the degree of IL- $1 \beta$ induced was low. Gene knockout of IL-6 rescued hypertension and hypertrophy in an angiotensin-II treatment for mice. ${ }^{49}$ In a pressure-overload study, TNF- $\alpha$ knockout partially rescued cardiac fibrosis and resulted in a normal ejection fraction. ${ }^{50}$ Despite the success in animal models, clinical trials and human studies have been mixed when targeting specific cytokines. Trials with IL- $1 \beta$ receptor antagonists improved exercise tolerance in heart failure ${ }^{51}$ and better outcomes post-acute MI. While these are encouraging, 
sample sizes were small ( $n=14$ and $n=30$, respectively) and should be repeated on a larger scale. Treatment in heart failure patients with TNF- $\alpha$ inhibitor improved cardiac function and remodeling in one trial ${ }^{52}$ but failed to show improved outcomes in another at similar doses and had a detrimental effect on patients at the higher dose. ${ }^{53}$ Numerous drugs targeting IL-6 or its inhibitor have passed clinical trials or are in clinical trials presently. However, none of these drugs have been tested directly in CVD. Instead, they are used in rheumatoid arthritis and cancer treatments. ${ }^{54}$

The therapeutic efficacy of single cytokine-specific intervention is unproven, likely due to functional redundancy with other cytokines. CARD9, on the other hand, appears to be necessary for a full innate immune response. A cursory glance at the literature will reveal that these pathways are rife with interest for scientists investigating inflammatory bowel syndrome, Crohn's disease, autoimmune disorders, and cancer. However, relatively little interest has been shown by those studying cardiometabolic and cardiovascular disorders. A few noteworthy exceptions exist, which we summarize here.

\section{Current research in CARD9- mediated vascular disease}

In a murine model, $\mathrm{CARD}^{-/}$protected against high-fat diet (HFD)-associated fibrosis and diminished fractional shortening, despite similar cardiac geometry compared to the wild type (WT). ${ }^{55}$ HFD induced myocardial infiltration by $\mathrm{CD} 68^{+}$immune cells. It also increased CARD9 protein expression and activation of 38 MAPK in the heart tissue and isolated macrophages. Activation of p38 MAPK was rescued in $\mathrm{CARD}^{-/-}$mice. In this model, CARD9 may potentiate inflammatory effects in part through its binding with NOD2, an intracellular PRR normally activated by bacteria pathogens. TNF- $\alpha$ and IL- 6 in the heart and isolated macrophages were elevated in the WT HFD group and restored to normal in the CARD9 $9^{-/}$HFD group. A similar pattern emerged for IL- $1 \beta$ in macrophages. IL- $1 \beta$ was not affected by diet or genotype. This does not preclude its attenuation from being in part responsible for protection, however, because IL-1 $\beta$ expression was still significantly lower in $\mathrm{CARD9}^{-/-}$mice. Furthermore, the cytokine profile was still generally elevated by HFD feeding. These findings are supported by the experiments of Ren et $\mathrm{al}^{56}$ who found that CARD9 ${ }^{-/-}$reduced fibrosis induced by infusion of angiotensin-II. Our work provides evidence that CARD9 plays a critical role in the development of cardiac hypertrophy, dysfunction, and fibrosis, all of which progress to heart failure. Accordingly, we are currently investigating the effect of transverse constriction of thoracic aorta (TAC) in CARD9 ${ }^{-/-}$mice.

Investigations in the NOD2 pathway provide indirect evidence that CARD9 may be an effective therapeutic target in atherosclerosis. For example, NOD2 ${ }^{-/-}$bone marrow transplantation into low-density lipoprotein receptor (LDLr) knockout mice reduced the size of the lipid-rich necrotic area in an aortic atherosclerotic lesion compared to WT bone marrow transplantation. ${ }^{57}$ However, transplant of RIP2 $2^{-/}$bone marrow had no effect. NOD2 also mediated oxidized LDL uptake by macrophages when stimulated by MDP. In another study, $\mathrm{RIP}^{-/-}$bone marrow transplantation simultaneously increased macrophage lipid uptake and exacerbated atherosclerosis in apolipoprotein B (APOB100XLDLr $\left.{ }^{-/}\right) .{ }^{58}$ As mentioned previously, NOD2 complexes with and signals through both RIP2 and CARD9. This finding and another showing CARD9 can mediate macrophage infiltration in smooth muscle cells ${ }^{59}$ suggest that CARD9 and RIP2 could play differential roles for NOD2 signaling in atherosclerosis. Interestingly, CARD9 and RIP2 operate in parallel downstream of SYK as well, which can associate with multiple PRRs, including dectin- ${ }^{14}$ and toll-like receptor 4 (TLR4). ${ }^{60,61}$

\section{Future research focuses}

CARD9 regulates multiple cytokines implicated in CVDs, but does not present with an obvious phenotype. It also does not require complete ablation of macrophages or neutrophils in order to be targeted. For these reasons, it serves as a valuable target for pharmacological intervention.

Experiments investigating the related inflammatory proteins NOD2 ${ }^{62}$ and NLR family, pyrin domain containing 3 (NLRP3) inflammasome ${ }^{63}$ signals that implicate them as potential targets ${ }^{57,58}$ are encouraging as CARD9 is the downstream effector for both pathways. ${ }^{19,64}$ Any benefit from the targeting of PRRs NOD2, NLRP3, dectin-1, or TLR4 may be included and compounded by targeting CARD9 instead. Furthermore, targeting CARD9 in lieu of NOD2 in atherosclerosis may leave beneficial RIP2 signaling intact. However, more research is necessary. CARD9 is under investigated in CVD and is thus highlighted for further study.

Future studies should begin by investigating the role of CARD9 in hypertensive heart disease using TAC models as well as atherosclerotic models, as macrophages and cytokines are complicit in plaque formation as well as myocardial remodeling.

Once protective effects of knockout are established in relevant models, care should be taken to ascertain which pathways are actually active in disease (and thus which are 
affected by treatment) and by what mechanism CARD9 is activated in a given disease model. This may help elucidate alternative pathways for treatment should serious side effects of CARD9 treatment be identified.

Despite the promising preliminary findings of cytokine inhibition, consistently effective pharmaceutical intervention remains elusive for inflammation-associated CVDs. It makes sense, then, to investigate proteins that may mediate more than one of these cytokine-mediating pathways simultaneously in order to produce a more protective cumulative effect. CARD9 is active in several inflammatory signaling pathways and non-redundantly so in some. As systemic and paracrine inflammations are present in several cardiovascular maladies, CARD9 inhibition presents an opportunity and should be targeted for research in CVD.

\section{Acknowledgements}

This publication was made possible by an Institutional Development Award (IDeA) from the National Institute of General Medical Sciences of the National Institutes of Health under Grant \# 2P20GM103432. Its contents are solely the responsibility of the authors and do not necessarily represent the official views of NIH.

\section{Disclosure}

The authors report no conflicts of interest in this work.

\section{References}

1. Nwanko T, Yun SS, Burt V, Gu Q. Hypertension among adults in the United States National Health and Nutrition Examination Survey, 2011-2012. NCHS Data Brief. 2013;2013(133):7.

2. Mozaffarian D, Benjamin EJ, Go AS, et al. Heart disease and stroke statistics-2016 update: a report from the American Heart Association. Circulation. 2016;133(4):e38-e360.

3. Yamaguchi O, Higuchi Y, Hirotani S, et al. Targeted deletion of apoptosis signal-regulating kinase 1 attenuates left ventricular remodeling. Proc Natl Acad Sci U S A. 2003;100(26):15883-15888.

4. Fink LN, Costford SR, Lee YS, et al. Pro-inflammatory macrophages increase in skeletal muscle of high fat-fed mice and correlate with metabolic risk markers in humans. Obesity (Silver Spring). 2014;22(3): 747-757.

5. Jheng HF, Tsai PJ, Guo SM, et al. Mitochondrial fission contributes to mitochondrial dysfunction and insulin resistance in skeletal muscle. Mol Cell Biol. 2012;32(2):309-319.

6. Li ZL, Woollard JR, Ebrahimi B, et al. Transition from obesity to metabolic syndrome is associated with altered myocardial autophagy and apoptosis. Arterioscler Thromb Vasc Biol. 2012;32(5):1132-1141.

7. Sosnovik DE, Nahrendorf M, Deliolanis N, et al. Fluorescence tomography and magnetic resonance imaging of myocardial macrophage infiltration in infarcted myocardium in vivo. Circulation. 2007;115(11): 1384-1391.

8. Kumar H, Kawai T, Akira S. Pathogen recognition by the innate immune system. Int Rev Immunol. 2011;30(1):16-34.

9. Bertin J, Guo Y, Wang L, et al. CARD9 is a novel caspase recruitment domain-containing protein that interacts with BCL10/CLAP and activates NF-kappa B. J Biol Chem. 2000;275(52):41082-41086.
10. Gross $\mathrm{O}$, Gewies A, Finger K, et al. Card9 controls a non-TLR signalling pathway for innate anti-fungal immunity. Nature. 2006;442(7103): 651-656.

11. Jia XM, Tang B, Zhu LL, et al. CARD9 mediates Dectin-1-induced ERK activation by linking Ras-GRF1 to H-Ras for antifungal immunity. J Exp Med. 2014;211(11):2307-2321.

12. Whibley N, Jaycox JR, Reid D, et al. Delinking CARD9 and IL-17: CARD9 protects against Candida tropicalis infection through a TNFalpha-dependent, IL-17-independent mechanism. J Immunol. 2015; 195(8):3781-3792.

13. Zhao XQ, Zhu LL, Chang Q, et al. C-type lectin receptor dectin-3 mediates trehalose 6,6'-dimycolate (TDM)-induced Mincle expression through CARD9/Bcl10/MALT1-dependent nuclear factor (NF)-kappaB activation. J Biol Chem. 2014;289(43):30052-30062.

14. Rogers NC, Slack EC, Edwards AD, et al. Syk-dependent cytokine induction by Dectin-1 reveals a novel pattern recognition pathway for C type lectins. Immunity. 2005;22(4):507-517.

15. Brown GD, Taylor PR, Reid DM, et al. Dectin-1 is a major $\beta$-glucan receptor on macrophages. J Exp Med. 2002;196(3):407-412.

16. Gow NA, Netea MG, Munro CA, et al. Immune recognition of Candida albicans beta-glucan by dectin-1. J Infect Dis. 2007;196(10): $1565-1571$.

17. Inohara N, Ogura Y, Fontalba A, et al. Host recognition of bacterial muramyl dipeptide mediated through NOD2. Implications for Crohn's disease. J Biol Chem. 2003;278(8):5509-5512.

18. Parkhouse R, Boyle JP, Mayle S, Sawmynaden K, Rittinger K, Monie TP. Interaction between NOD2 and CARD9 involves the NOD2 NACHT and the linker region between the NOD2 CARDs and NACHT domain. FEBS Lett. 2014;588(17):2830-2836.

19. Hsu YM, Zhang Y, You Y, et al. The adaptor protein CARD9 is required for innate immune responses to intracellular pathogens. Nat Immunol. 2007;8(2):198-205.

20. Seth RB, Sun LJ, Ea CK, Chen ZJJ. Identification and characterization of MAVS, a mitochondrial antiviral signaling protein that activates NF-kappa B and IRF3. Cell. 2005;122(5):669-682.

21. Poeck H, Bscheider M, Gross O, et al. Recognition of RNA virus by RIG-I results in activation of CARD9 and inflammasome signaling for interleukin 1 beta production. Nat Immunol. 2010;11(1):63-69.

22. Abdullah Z, Schlee M, Roth S, et al. RIG-I detects infection with live Listeria by sensing secreted bacterial nucleic acids. EMBO J. 2012;31(21):4153-4164.

23. Glocker EO, Hennigs A, Nabavi M, et al. A homozygous CARD9 mutation in a family with susceptibility to fungal infections. $N$ Engl $J$ Med. 2009;361(18):1727-1735.

24. Nakamura S, Nakamura S, Matsumoto T, et al. Overexpression of caspase recruitment domain (CARD) membrane-associated guanylate kinase 1 (CARMA1) and CARD9 in primary gastric B-cell lymphoma. Cancer. 2005;104(9):1885-1893.

25. Yang M, Shao JH, Miao YJ, et al. Tumor cell-activated CARD9 signaling contributes to metastasis-associated macrophage polarization. Cell Death Differ. 2014;21(8):1290-1302.

26. Zhernakova A, Festen EM, Franke L, et al. Genetic analysis of innate immunity in Crohn's disease and ulcerative colitis identifies two susceptibility loci harboring CARD9 and IL18RAP. Am J Hum Genet. 2008; 82(5):1202-1210.

27. Lamas B, Richard ML, Leducq V, et al. CARD9 impacts colitis by altering gut microbiota metabolism of tryptophan into aryl hydrocarbon receptor ligands. Nat Med. 2016;22(6):598-605.

28. Lee EJ, Brown BR, Vance EE, et al. Mincle activation and the Syk/ Card9 signaling axis are central to the development of autoimmune disease of the eye. J Immunol. 2016;196(7):3148-3158.

29. Yasukawa S, Miyazaki Y, Yoshii C, et al. An ITAM-Syk-CARD9 signalling axis triggers contact hypersensitivity by stimulating IL-1 production in dendritic cells. Nat Commun. 2014;5:3755.

30. Yang ZW, Weng CZ, Wang J, Xu P. The role of Card9 overexpression in peripheral blood mononuclear cells from patients with aseptic acute pancreatitis. J Cell Mol Med. 2016;20(3):441-449. 
31. Espinola-Klein C, Rupprecht HJ, Blankenberg S, et al. Impact of infectious burden on progression of carotid atherosclerosis. Stroke. 2002;33(11):2581-2586.

32. Danesh J, Whincup P, Walker M, et al. Low grade inflammation and coronary heart disease: prospective study and updated meta-analyses. Br Med J. 2000;321(7255):199-204.

33. Frangogiannis NG. Chemokines in the ischemic myocardium: from inflammation to fibrosis. Inflamm Res. 2004;53(11):585-595.

34. Azzawi M, Kan SW, Hillier V, Yonan N, Hutchinson IV, Hasleton PS. The distribution of cardiac macrophages in myocardial ischaemia and cardiomyopathy. Histopathology. 2005;46(3):314-319.

35. Naruko T, Ueda M, Haze K, et al. Neutrophil infiltration of culprit lesions in acute coronary syndromes. Circulation. 2002;106(23):2894-2900.

36. Chandrasekar B, Smith JB, Freeman GL. Ischemia-reperfusion of rat myocardium activates nuclear factor-kappa $\mathrm{B}$ and induces neutrophil infiltration via lipopolysaccharide-induced CXC chemokine. Circulation. 2001;103(18):2296-2302.

37. Morimoto H, Takahashi M, Izawa A, et al. Cardiac overexpression of monocyte chemoattractant protein-1 in transgenic mice prevents cardiac dysfunction and remodeling after myocardial infarction. Circ Res. 2006;99(8):891-899.

38. Frangogiannis NG, Dewald O, Xia Y, et al. Critical role of monocyte chemoattractant protein-1/CC chemokine ligand 2 in the pathogenesis of ischemic cardiomyopathy. Circulation. 2007;115(5):584-592.

39. Zandbergen HR, Sharma UC, Gupta S, et al. Macrophage depletion in hypertensive rats accelerates development of cardiomyopathy. J Cardiovasc Pharmacol Ther. 2009;14(1):68-75.

40. Kain D, Amit U, Yagil C, et al. Macrophages dictate the progression and manifestation of hypertensive heart disease. Int J Cardiol. 2016;203: 381-395.

41. Ma FF, Li YL, Jia LX, et al. Macrophage-stimulated cardiac fibroblast production of IL-6 is essential for TGF beta/Smad activation and cardiac fibrosis induced by angiotensin II. PLoS One. 2012;7(5):e35144.

42. Melendez GC, McLarty JL, Levick SP, Du Y, Janicki JS, Brower GL. Interleukin 6 mediates myocardial fibrosis, concentric hypertrophy, and diastolic dysfunction in rats. Hypertension. 2010;56(2):225-231.

43. Palmer JN, Hartogensis WE, Patten M, Fortuin FD, Long CS. Interleukin-1beta induces cardiac myocyte growth but inhibits cardiac fibroblast proliferation in culture. J Clin Investig. 1995;95(6):2555-2564.

44. LeibundGut-Landmann S, Gross O, Robinson MJ, et al. Syk- and CARD9dependent coupling of innate immunity to the induction of Thelper cells that produce interleukin 17. Nat Immunol. 2007;8(6):630-638.

45. Liu YF, Zhu HT, Su ZL, et al. IL-17 contributes to cardiac fibrosis following experimental autoimmune myocarditis by a PKC beta/Erk1/2/NF-kappa B-dependent signaling pathway. Int Immunol. 2012;24(10):605-612.

46. Van Tassell BW, Arena RA, Toldo S, et al. Enhanced interleukin-1 activity contributes to exercise intolerance in patients with systolic heart failure. PLoS One. 2012;7(3):e33438.

47. Bozkurt B, Kribbs SB, Clubb FJ, et al. Pathophysiologically relevant concentrations of tumor necrosis factor-alpha promote progressive left ventricular dysfunction and remodeling in rats. Circulation. 1998;97(14): 1382-1391.

48. Honsho S, Nishikawa S, Amano K, et al. Pressure-mediated hypertrophy and mechanical stretch induces IL-1 release and subsequent IGF-1 generation to maintain compensative hypertrophy by affecting Akt and JNK pathways. Circ Res. 2009;105(11):U1149-U1265.
49. Sun M, Chen MY, Dawood F, et al. Tumor necrosis factor-alpha mediates cardiac remodeling and ventricular dysfunction after pressure overload state. Circulation. 2007:115(11):1398-1407.

50. Coles B, Fielding CA, Rose-John S, Scheller J, Jones SA, O’Donnell VB. Classic interleukin-6 receptor signaling and interleukin-6 transsignaling differentially control angiotensin II-dependent hypertension, cardiac signal transducer and activator of transcription-3 activation, and vascular hypertrophy in vivo. Am J Pathol. 2007;171(1):315-325.

51. Van Tassell BW, Arena R, Biondi-Zoccai G, et al. Effects of interleukin-1 blockade with anakinra on aerobic exercise capacity in patients with heart failure and preserved ejection fraction (from the D-HART Pilot Study). Am J Cardiol. 2014;113(2):321-327.

52. Bozkurt B, Torre-Amione G, Warren MS, et al. Results of targeted antitumor necrosis factor therapy with etanercept (ENBREL) in patients with advanced heart failure. Circulation. 2001;103(8):1044-1047.

53. Chung ES, Packer M, Lo KH, Fasanmade AA, Willerson JT, Investigators A. Randomized, double-blind, placebo-controlled, pilot trial of infliximab, a chimeric monoclonal antibody to tumor necrosis factor-alpha, in patients with moderate-to-severe heart failure - results of the anti-TNF therapy against congestive heart failure (ATTACH) trial. Circulation. 2003;107(25):3133-3140.

54. Kim GW, Lee NR, Pi RH, et al. IL-6 inhibitors for treatment of rheumatoid arthritis: past, present, and future. Arch Pharm Res. 2015; 38(5):575-584.

55. Cao L, Qin X, Peterson MR, et al. CARD9 knockout ameliorates myocardial dysfunction associated with high fat diet-induced obesity. J Mol Cell Cardiol. 2016;92:185-195.

56. Ren J, Yang M, Qi G, et al. Proinflammatory protein CARD9 is essential for infiltration of monocytic fibroblast precursors and cardiac fibrosis caused by Angiotensin II infusion. Am J Hypertens. 2011;24(6):701-707.

57. Johansson ME, Zhang XY, Edfeldt K, et al. Innate immune receptor NOD2 promotes vascular inflammation and formation of lipid-rich necrotic cores in hypercholesterolemic mice. Eur J Immunol. 2014; 44(10):3081-3092.

58. Levin MC, Jirholt P, Wramstedt A, et al. Rip2 deficiency leads to increased atherosclerosis despite decreased inflammation. Circ Res. 2011; 109(11):1210-1218.

59. Liu Y, Wang Y, Shi H, et al. CARD9 mediates necrotic smooth muscle cell-induced inflammation in macrophages contributing to neointima formation of vein grafts. Cardiovasc Res. 2015;108(1):148-158.

60. Miller YI, Choi SH, Wiesner P, Bae YS. The SYK side of TLR4: signalling mechanisms in response to LPS and minimally oxidized LDL. Br J Pharmacol. 2012;167(5):990-999.

61. Phongsisay V, Iizasa E, Hara H, Yoshida H. Evidence for TLR4 and FcRgamma-CARD9 activation by cholera toxin $\mathrm{B}$ subunit and its direct bindings to TREM2 and LMIR5 receptors. Mol Immunol. 2015;66(2): 463-471.

62. Liu Y, Yang H, Liu LX, et al. NOD2 contributes to myocardial ischemia/ reperfusion injury by regulating cardiomyocyte apoptosis and inflammation. Life Sci. 2016;149:10-17.

63. Kawaguchi M, Takahashi M, Hata T, et al. Inflammasome activation of cardiac fibroblasts is essential for myocardial ischemia/reperfusion injury. Circulation. 2011;123(6):594-605.

64. Gross O, Poeck H, Bscheider M, et al. Syk kinase signalling couples to the Nlrp3 inflammasome for anti-fungal host defence. Nature. 2009; 459(7245):433-436.
Drug Design, Development and Therapy

\section{Publish your work in this journal}

Drug Design, Development and Therapy is an international, peerreviewed open-access journal that spans the spectrum of drug design and development through to clinical applications. Clinical outcomes, patient safety, and programs for the development and effective, safe, and sustained use of medicines are the features of the journal, which
Dovepress

has also been accepted for indexing on PubMed Central. The manuscript management system is completely online and includes a very quick and fair peer-review system, which is all easy to use. Visit http://www.dovepress.com/testimonials.php to read real quotes from published authors. 